Title:

\title{
Emerging Pathogens: Dynamics, Mutation and Drug Resistance
}

Mutation and Drug Resistance

Authors:

Submitted to:

A. S. Perelson, $T-10$

PECEIVED

B. Goldstein, T-10

B. T. Korber, T-10

OCT $O 11997$

C. A. Macken, T-10

O.STI

N. I. Stilianakis, $T-10$

F. G. Hayden, U. Virginia

DOE Office of Scientific and Technical Information (OSTI)

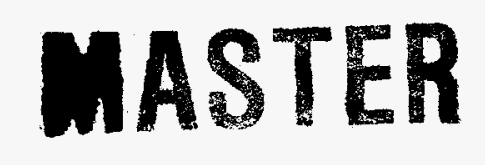

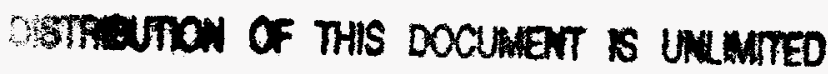

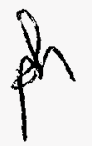

\section{Los Alamos}

NATIONAL LABORATORY

Los Alamos National Laboratory, an affirmative action/equal opponunity employer, is operated by the University of California for the U.S. Department of Energy under contract W-7405-ENG-36. By acceptance of this article, the publisher recognizes that the U.S. Government retains a nonexclusive, royattyfree license to publish or reproduce the published Iorm of this contribution, or to allow others to do so, for U.S. Government purposes. Los Alamos National Laboratory requests that the publisher identity this article as work periormed under the auspices of the U.S. Department of Energy. Los Alamos Nattonal Laboratory strongly supports academic freedom and a researcher's right to publish; as an institution, however, the Laboratory does not endorse the viewpoint of a publication or guarantee its technical correctness. 


\section{DISCLAIMER}

This report was prepared as an account of work sponsored by an agency of the United States Government. Neither the United States Government nor any agency thereof, nor any of their employees, make any warranty, express or implied, or assumes any legal liability or responsibility for the accuracy, completeness, or usefulness of any information, apparatus, product, or process disclased, or represents that its use would not infringe privately owned rights. Reference herein to any specific commercial product, process, or service by trade name, trademark, manufacturer, or otherwise does not necessarily constitute or imply its endorsement, recommendation, or favoring by the United States Government or any agency thereof. The views and opinions of authors expressed herein do not necessarily state or reflect those of the United States Government or any agency thereof. 


\section{DISCLAMIER}

Portions of this document may be illegible in electronic inage products. Images are produced from the best avaliable original document. 


\title{
Emerging Pathogens: Dynamics, Mutation and Drug Resistance
}

\author{
Alan S. Perelson, Byron Goldstein, Bette T. Korber, \\ Catherine A. Macken, and Nikolaos I. Stilianakis \\ Los Alamos National Laboratory \\ Frederick G. Hayden \\ University of Virginia
}

\begin{abstract}
This is the final report of a one-year, Laboratory Directed Research and Development (LDRD) project at Los Alamos National Laboratory (LANL). The objectives of this project were to develop models of the spread of pathogens, such as HIV-1 and influenza, in humans, and then to use the models to address the possibility of designing appropriate drug therapies that may limit the ability of the pathogen to escape treatment by mutating into a drug resistant form. We have developed a model of drug-resistance to amantidine and rimantadine, the two major antiviral drugs used to treat influenza, and have used the model to suggest treatment strategies during an epidemic.
\end{abstract}

\section{Background and Research Objectives}

Pathogens that can grow rapidly and mutate into drug resistant forms pose a serious health threat to the US and the world-wide population. In the case of HIV-1, potent new drugs are being developed, which if applied appropriately, may be able to eradicate the virus. In the case of influenza, two antiviral drugs, amantadine and rimantadine are currently available and in limited use. Influenza is one of the world's greatest killers. In a normal flu season between 20,000 and 40,000 deaths in the US are attributable to influenza infection. In the 1918--1919 pandemic, over 21 million people world-wide died. The use of anti-viral drugs should be able to prevent such large numbers of deaths if another pandemic strikes.

Drug treatment during influenza virus infection has been associated with the emergence of drug resistant viruses. Individuals infected with influenza shed resistant viruses two to five days into treatment with the antiviral drugs amantadine and rimantadine. The impact of the emergence of drug-resistant viruses and the likelihood of their transmission is unknown. Since in most circumstances the benefits of drug prophylaxis and therapy outweigh the risks of drug-resistant influenza viruses emerging, drug treatment is one considered way of providing prevention and therapy of influenza infections.

- Principal Investigator, Email: asp@t10.lanl.gov 
The health significance of the emergence of drug-resistant viruses needs to be addressed before large-scale treatment programs are begun, as for example might occur in a major outbreak or pandemic. One needs to determine strategies for treatment that would keep emergence of drug-resistant viruses at reasonably low levels. At the same time, a good strategy should still allow prevention and treatment of infected individuals to a reasonable extent.

\section{Importance to LANL's Science and Technology Base and National R\&D Needs}

The Laboratory has a core competency in Bioscience and Biotechnology, which includes capabilities in theoretical and computational biology. In addition, there are now growing national concerns about the use of biological agents for terrorist activities as well as in warfare. The work that we have embarked on may be of great significance to both of these programs. It enhances our basic modeling strengths, as part of our core competency, and it enhances our ability to deal with biological threats. Influenza, besides its potential use as a biological agent in germ-warfare, can be used as a model for the spread of other highly contagious, airborne pathogens. The information that we gain from modeling influenza spread and therapy may be applicable to a variety of other infectious agents that could be released into populations by terrorists or as part of germ warfare.

\section{Scientific Approach and Accomplishments}

Antiviral treatment with amantadine and rimantadine has been associated with the emergence of drug-resistant influenza A viruses [1 - 3]. Such variants are fully resistant to the selective antiviral effects of clinically observable drug concentrations [4]. Furthermore, in animal models of influenza the prophylactic activity of amantadine and rimantadine are completely lost when animals are exposed to resistant virus. Drug-treated persons infected with influenza may shed resistant viruses within two to five days of starting treatment [1, 3]. The impact of the emergence of drug-resistant viruses and the likelihood of their transmission are incompletely defined, although transmission of such variants has been associated with failures of drug prophylaxis and therapy in households and nursing homes $[1-3,5]$. In most circumstances the benefits of drug prophylaxis and therapy appear to outweigh the risks of drug resistance emergence [4]. 
The epidemiologic significance of the emergence of drug-resistant viruses needs to be addressed before recommendations for large-scale drug-administration programs are made. One needs to determine strategies for treatment that would keep emergence of drugresistant viruses at reasonably low levels and minimize the deleterious effects of such viral variants. If possible, such strategies should still allow prevention of illness and treatment of ill patients to take maximal advantage of the proven prophylactic and therapeutic activities of these antiviral agents.

We have developed a mathematical model that describes the effects of drug resistance on the transmission dynamics of an influenza epidemic/outbreak in a closed population. Using the model, several drug administration strategies have been considered and evaluated with respect to the degree to which the drug treatment and/or prophylaxis impacts on the emergence of drug resistance. The influence of prior influenza immunization on estimating the protective effect of the drug has also been addressed.

\section{Modeling an Outbreak}

Strategies for administering antiviral therapy during an influenza epidemic have been studied by modeling the epidemic using a susceptible-infective-removed (SIR) model in which the total population remains constant and is homogeneously mixed. The assumption of a constant population is reasonable if the infection spreads quickly through the population, which is the case in an influenza epidemic. Further, we have assumed that deaths and the time between exposure and the beginning of infectiousness are negligible. Also, we have ignored high-risk groups. To demonstrate the validity of the model we have fit data from a previous influenza outbreak that occurred in a single wave [6].

The best example of modeling an influenza epidemic in a closed population is the situation of a school or a nursing home where the susceptibles can be assumed to represent an "idealized" closed population. For that purpose we employed the data of an influenza A epidemic that took place from January 15 to February 15, 1978 in a boarding school [6]. We modeled the influenza epidemic with a simple but conceptually new SIR model where, in contrast to the classical SIR approach, we distinguished between two classes of infected and therefore infective individuals: infected asymptomatic individuals (subclinical infections) and infected individuals developing clinical symptoms (illness). The reason we made this distinction between infecteds is that there are likely differences in disease transmission between these subgroups. In addition, studies conducted to determine the effects of the use of amantadine prophylaxis on influenza infections [7-11] indicate that subclinical infections accounted for approximately one-third of infections. 
Since not every infection leads to illness and prophylaxis raises the probability of having a subclinical infection [7-11], therapy and/or prophylaxis cause a redistribution of individuals among the groups of infecteds. An infected individual with clinical symptoms sheds more virus than an individual with a subclinical infection. On the other hand, infected individuals having clinical symptoms may show reduced viral transmissibility if they are sufficiently ill to be confined to bed. Hence, the magnitude of the difference between the two transmission rates depends on factors like age, severity of illness, social behavior, and design of living quarters (e.g. common rooms, shared bedrooms). Although the splitting of infected individuals into two classes might be in some cases redundant from the dynamic point of view, it is reasonable to keep this distinction on biological grounds and it is useful in analyzing the effects of drug treatment.

As in the standard SIR model we also considered a class of uninfected susceptible individuals and a class of the infecteds who recover from either subclinical or clinical infections during the epidemic. Transmission of infection results from contacts of the susceptible population with any of the above classes of infected individuals. Infection of a susceptible initially leads to subclinical infection (incubation period). Subclinically infected individuals either develop clinical symptoms and then recover or recover before developing clinical symptoms. Transition from the asymptomatic-infected to the clinically-infected stage occurs for individuals who do not recover before developing clinical symptoms. All infecteds, whether subclinically infected or with symptoms, eventually recover from infection.

We assumed that the epidemic starts in a closed population of susceptibles with the introduction of a few infected individuals that show clinical symptoms; the subclinical cases which might precede the sick ones are not detectable. Since the epidemic we modeled spread within the school population [6], the basic reproduction number, i.e., the number of secondary cases caused during the epidemic by an infected individual in a completely susceptible population, was above its critical value of one.

\section{Modeling Effects of Drug Administration}

The model we developed is considerably more complex than the standard SIR model, because we considered the effects of drug given either prophylactically, i.e., before infection occurs, or as a treatment, i.e., after infection has occurred, and the generation of drug-resistant viral variants. Thus, the model depicted in Fig. 1 considered the following populations: susceptibles (S), susceptibles taking drug prophylactically (SP), infecteduntreated (IU), infected-untreated developing clinical symptoms (IUS), infected-untreated asymptomatic shedding drug resistant virus (IUR), infected-untreated with clinical 
symptoms shedding drug resistant virus (IUSR), infected-treated (IT), infected-treated developing clinical symptoms (ITS), infected-treated asymptomatic shedding drug resistant virus (ITR), and infected-treated with clinical symptoms shedding resistant virus, (ITSR).

In surveys of influenza A virus isolates recovered from untreated patients, less than $1 \%$ have been drug-resistant [12]. Although such viruses might represent naturally resistant variants, we assumed that resistant influenza variants are not present at the time the epidemic is recognized. The existence of naturally resistant variants can be easily included in the model by using a positive value for the initial condition of the IUR group.

Identification of an epidemic typically occurs when about $1--5 \%$ of the considered population has been infected. Drug-resistant viruses emerge rapidly after the beginning of treatment (2--5 days) [1, 3]. Hence, we modeled the epidemic as a continuous process starting at the time when 1--5\% of the population was infected and illness was detected. We also assumed that treatment and/or prophylaxis can begin at this time point.

Transmission of infection is assumed to occur with a rate $\beta$, and results from contacts of the susceptible population, whether taking prophylaxis or not, with any of the above classes of infected individuals. Infection of a susceptible initially leads to subclinical infection (incubation period). Subclinically infected individuals either develop clinical symptoms and then recover or recover before developing clinical symptoms. Transition from the asymptomatic-infected to the clinically-infected stage is assumed to occur with a rate $\delta$. All infecteds, whether subclinically infected or with symptoms, recover with some rate $\gamma$. The rate of development of drug resistance is $\kappa$. Finally, the rate of receiving treatment and/or prophylaxis is $\theta$ (see Fig. 1).

The transmission rate between a susceptible and an IUS individual is much higher than that of a susceptible and an IU person. Susceptibles are assumed to get infected by IU individuals with the same rate as by IUR and ITR individuals (i.e. in those shedding resistant virus, drug treatment does not prolong or reduce viral replication compared to no treatment and resistant virus is assumed to be as infective as wild type virus).

Drug treatment reduces disease severity and the probability of transmitting the wild type disease. We assumed that the reduction of infectivity, and therefore the risk of infection, in an encounter of a susceptible with an IT is about $60-70 \%$ of that an encounter with an IU person. The reduction of infectivity, and therefore the risk of clinical infection, in an encounter of a susceptible with an ITS person is assumed to be about $90 \%$ of that of an encounter with an IUS person. This is based on the observed antiviral effects of drug treatment in ill adults [13] and the reduced number of secondary cases due to 
household contacts when ill index cases are given drug treatment [14]. These estimates, however, account for the situation of an epidemic where variable degrees of prior immunity exist. In a pandemic, due to no prior immunity, the corresponding estimates are probably lower.

Once drug resistance develops, treatment has little effect. Therefore, we assumed that there is no difference in transmission through contacts between susceptibles and infecteds with clinical symptoms shedding resistant virus whether treated (ITSR) or not (IUSR). Further, we assumed resistant virus is transmitted as easily as wild type virus. These assumptions are based on direct observations in experimental avian influenza [15].

Prophylaxis is assumed to reduce the susceptibility of contacts [6-11]. The transmission rate of IU individuals to an SP individual, is about $2 / 3$ of the corresponding rate to a susceptible who does not take prophylaxis. This assumption is based on results of amantadine prophylaxis studies during pandemic influenza [7-11]. The corresponding value for the case of an epidemic, due to some degree of prior immunity, is assumed to be lower (by about 1/3). Furthermore, the reduction of transmission from IUS to SP individuals is expected to be larger (say, $60-70 \%$ reduction for a pandemic and $90 \%$ reduction for an epidemic). The same assumption applies for ITS individuals having contacts with SP individuals. The probability that an IT or an ITS persons can infect an SP is assumed to be very small ( 0.1 and 0.01 relative infectivity of the wild type during treatment/prophylaxis compared with that without drug intervention during an epidemic). SP individuals who encounter ITR and IUR individuals are assumed to be minimally protected and have the same transmission rates as their counterparts that do not take prophylaxis. In the cases of ITSR or IUSR persons these rates are also the same as the corresponding ones from the subclinical cases.

The above estimates about the transmission rates come from several studies conducted during influenza outbreak situations where treatment and emergence of drug resistant strains were observed [1-4]. Despite the fact that the prophylactic efficacy of amantadine against infection ranges between $19 \%$ and $52 \%$ in pandemics [7-11], most studies deal with populations that have been naturally infected with related viruses or immunized prior to the epidemic. Under those circumstances the prophylactic efficacy for preventing infection or illness are higher. Consequently, we have assumed that an SP individual who encounters an IU, IUS, IT or ITS individual is better protected than in the estimates of the studies derived from prophylaxis during pandemics.

We have also employed a second set of transmission rates which supports the assumption that drug-resistant influenza strains developed during treatment or transmitted to susceptibles from infecteds under treatment are less infectious than the wild type 
influenza virus. Therefore, susceptibility and infectivity between susceptible and infected individuals is lowered significantly for resistant strains. Infectivity among infecteds that shed resistant virus (IUR, IUSR, ITR, ITSR) and have contacts with susceptible or SP persons is assumed to be reduced by $90 \%$ in both cases, which corresponds to the infectivity level of subclinical infection with the wild type virus (in the case of IUSR and ITSR persons) or 10-fold less (in the case of IUR, ITR persons).

The recovery rate of infected individuals appears to be the same for illness due to wild type and drug-resistant virus $[1,16]$. Further, we have assumed that drug treatment does not enhance recovery of individuals shedding resistant virus. In individuals showing clinical symptoms these rates may be slightly different since those infecteds who shed drug-resistant virus, whether treated or not, may have a longer infectivity period [16, 17]. Infected individuals that have been treated are expected to recover faster. Individuals with clinical symptoms have a lower recovery rate than asymptomatic infecteds. This assumption does not take into account that infecteds with clinical symptoms are to some degree isolated since they feel poorly and tend to avoid contacts with susceptibles and/or susceptibles are more careful in their behavior to them. Thus, one could argue that it is more likely that their average period of infectivity (in the sense of removal from the population that transmits the disease) is shorter or almost the same as that of untreated infecteds. This behavior is probably outweighed by their higher infectivity due to higher viral loads and presence of symptoms.

The rates with which IU, IUR, IT and ITR individuals develop clinical symptoms are assumed to relate to each other such as treatment slows down the appearance of clinical symptoms (e.g. IT individuals become ITS with a much lower rate than IU become IUS). In untreated persons infected with wild type or resistant viruses and treated individuals shedding resistant virus, the development of symptoms occurs at the same rate.

Drug-resistant variants of influenza occur at very low levels in untreated populations. Thus, we have ignored the spontaneous generation of drug-resistant variants in untreated individuals during the course of a single outbreak. However, following treatment resistance is observed to develop. In the absence of clinical symptoms, we have assumed there is less viral replication and hence less of a chance of resistance developing.

\section{Drug Interventions}

Drug administration strategies can be subdivided into treatment of infected individuals or prophylaxis of susceptibles, or both. Further, treatment can be administered at some average rate during the epidemic or instantaneously at a certain time point. The latter would seem to apply only for cases of small populations, e.g., schools, nursing 
homes or hospitals, where rapid drug distribution to susceptible and infected individuals might be possible.

Drug administration strategies have been modeled by removal rates from one class to another. Susceptibles can receive prophylaxis with some daily rate. This rate may be time dependent and/or a function of the number of infected individuals availahle. Infected individuals, who shed drug resistant virus (IUR) or not (IU), upon treatment are removed from their class and put in the class of infected-treated individuals with some rate.

Obviously, treatment of an infected person (I) is possible only if their subclinical infective status is detected or if prophylaxis is given. IUS and IUSR individuals are also assumed to be treated with a rate that can be different for each of these groups. Although IUSR individuals will probably not respond to treatment, they are assumed to receive treatment because, they are not recognized prospectively as shedding resistant virus.

\section{Simple Epidemic Model}

Our basic model considered a single wave of an influenza epidemic in a closed population without deaths of infecteds. This model is based on a reported epidemic in a boarding school with 578 boys [6]. During the 30 day epidemic, 166 boys (28.7\% of total student number) developed influenzal illness. The average length of fever or other symptoms was 4 days. The number of subclinical infections was unknown. At day seven of the epidemic, 31 non-ill boys (5.4\% of total student population) received amantadine prophylaxis. No investigation was conducted with respect to the question of development of drug resistant influenza strains.

The boys had been immunized with an influenza vaccine that was antigenically different from the epidemic virus, and therefore it failed to prevent the epidemic [6]. Since our intention was to develop models useful in pandemic situations, we did not include in our model the possible effect of immunization. Thus, to examine this outbreak we make some modifications. The number of infecteds developing illness in influenza epidemics varies between $20 \%$ and $75 \%$. There is also a variable fraction of immune (nonsusceptible) persons in the population. We assumed that about $220(38 \%)$ of the 578 boys were totally immune to the influenza virus and therefore they were not affected during the epidemic. The remaining 378 boys formed our pool of susceptibles. This number of immunes is in line with the levels of immunity observed in a second report where amantadine was again used during an influenza outbreak in the year 1980 in the same school [18]. Further, this number gave the best fit for our data and lies within the estimates of possible immunes within a population. 
In order to fit the data, we assumed that an average of $50 \%$ become sick with clinical symptoms (and the remaining $50 \%$ to be subclinically infected). The ratio between the transmission rates of subclinically infected and clinically infected is assumed to be 1:10. We fit the data assuming that prophylaxis is given to 31 boys starting at day 7 . According to the data, 24 boys ( $4.15 \%$ of total student population) showed clinical symptoms at that day. Fig. 2 shows the close agreement between our model and the data obtained during this outbreak.

\section{Epidemic with Treatment}

In order to model the effects of drug administration in the above typical epidemic described in Figure 2, we introduced treatment at day 7, as was originally done [6], and followed the epidemic for 30 days. We compared the number of infecteds showing clinical symptoms with the typical epidemic described above (but without any intervention). The following three treatment and prophylaxis strategies were considered: 1) treatment of infected individuals showing clinical symptoms only; 2) prophylaxis of all susceptible individuals not having been infected by day seven; 3) treatment for infecteds as under 1) and strategy prophylaxis for all susceptibles as under 2).

Strategy 1: One plausible approach is treatment only of infecteds with clinical symptoms. We choose a per capita treatment rate of $0.5 /$ day for IUS, IUSR individuals, which implies that the time to treat half the population is 1.4 days. With this treatment rate there is a damping and at the same time a prolongation of the epidemic. The total number of infecteds with or without clinical symptoms remains almost the same as in the case without treatment. Figure $3 \mathrm{a}$ shows that after the introduction of treatment the fraction of ITS individuals (dotted line) continues to increase for about 5 days and then declines. This would reduce the epidemic significantly if no resistance developed. The model however predicts the initiation of a second epidemic caused by drug-resistant variants that emerge from the treatment of the infecteds (dashed line Fig. 3a). The emergence of resistant virus is responsible for the prolongation of the epidemic. The fraction of the infecteds with clinical symptoms who shed resistant virus after 30 days is $47.7 \%$ of the total number of sick individuals. The fraction of sick individuals is $49.2 \%$ of the initial susceptible population. Thus, under the assumption of equal infectivity between wild type and drugresistant virus, the model predicts larger numbers of infecteds with drug-resistant ivirus than have been observed.

Since the estimate of the drug efficacy during influenza epidemics is not precise, we also simulated the model with a second set of parameters that includes a reduction of $90 \%$ in infectivity of drug-resistant influenza strains transmitted from infected individuals that 
carry those strains. The results in Fig. $4 \mathrm{a}$ show that following the same treatment strategy there is a difference in the dynamic behavior of the infected population. The fraction of infecteds shedding resistant virus is now much lower $(29.9 \%)$ of the total number of ill individuals after 30 days. The fraction of ill individuals themselves is now lower, $41.1 \%$ of the initial number of susceptibles. The total number of infecteds is also reduced. This indicates that by assuming a reduction in the infectivity of drug-resistant strains there is benefit in treating only infecteds.

Strategy 2: Another simple strategy is to give prophylaxis to all susceptibles who have not yet been infected, that is, mass chemoprophylaxis for outbreak control. The distribution of the drug in our model is assumed to occur within a day. Prophylaxis is initiated at day seven. Figure $3 \mathrm{~b}$ shows that after a day the fraction of infected ill individuals goes down rapidly but again we predict emergence of resistant virus caused now by the individuals taking prophylaxis. The epidemic is predicted to last significantly longer than if no prophylaxis were given.

The number of infected ill individuals is after 30 days a bit lower than under strategy 1 . Yet, one has to take into account that the second epidemic wave caused by the drug-resistant variants is at day 30 still ongoing (Fig. 3b).

The assumption that there is no reduction in infectivity for drug-resistant virus allows the transmissibility to remain as high as before the initiation of prophylaxis for this group and this causes the second wave of the epidemic. Thus the model predicts that with prophylaxis the epidemic runs longer. However, the predicted observations shown in Fig. $3 \mathrm{~b}$ do not correlate with published experiences in using this strategy of mass chemoprophylaxis (with or without treatment of ill persons). Termination of the outbreak is usually found and the number of clinical prophylaxis failures is usually very low, even in studies reporting the emergence of resistant variants $[2,3,5]$. In such circumstances, clinical influenza has developed in $4-10 \%$ of patients receiving prophylaxis and proven prophylaxis failures due to resistant virus has occurred in $2-3 \%$ of prophylaxis recipients.

If we re-examine this scenario with a decrease of $90 \%$ in infectivity of drugresistant strains, the model predicts a fast damping of the epidemic with a small fraction of cases where infecteds shed drug resistant virus (Fig. 4b). The ill infecteds decline one day after the introduction of prophylaxis and after 20 days of the initiation of the epidemic there is almost no infected person. About $89.9 \%$ of the persons who received prophylaxis at day seven remain uninfected at day 30 . Only $14.8 \%$ of the initial susceptible population became ill. Moreover, the total number of infecteds with clinical symptoms having been infected during the period of 30 days is only about $29.9 \%$ of the cases in the original epidemic and the epidemic passes very fast. Cases of infected individuals that shed 
resistant virus make only $3.8 \%$ of the total number of ill individuals. These predictions are in line with the observations of the studies mentioned above.

Thus, if infectivity of drug-resistant variants is lower than that of the wild type virus, then prophylaxis offers high levels of protection and it would be a very successful prevention strategy.

Strategy 3: The third strategy that we have modeled involves combining prophylaxis with treatment of infected individuals, using the same assumptions as in strategies 1 and 2 . Figure $3 \mathrm{c}$ shows that this combined strategy gives similar results to those obtained for strategy 1 or 2 , with prolongation of the epidemic and a second epidemic wave caused by drug-resistant virus. For the same reasons as in strategy 1 , treatment favors drug resistance since the infectivity of individuals shedding drug-resistant virus is assumed to be the same as that of the wild-type virus. In addition, prophylaxis and/or treatment offers some protection to individuals in contact with infecteds shedding the wild type virus but no protection at all in the case of resistant virus. This gives an advantage to emergence and faster transmission of resistant virus, which replaces the wild type virus among infecteds. Also, in this case, the total number of infecteds is slightly less than the total number of infecteds in the original epidemic and there is no beneficial effect with respect to the total epidemic. In fact there are a few more cases of infecteds in total. The fraction of the sick infecteds shedding resistant virus among the total of the sick individuals is approximately $77 \%$.

The alternative scenario in which the infectivity of drug-resistant strains is $90 \%$ lower than the infectivity of the wild type virus, Fig. 4c, shows that the combined protective effects of prophylaxis and treatment forces the epidemic to run at very low levels giving a total number of sick infecteds that is $33.3 \%$ of the corresponding number in the original epidemic. The fraction of sick infecteds shedding resistant virus within this group is still high, i.e., $33.9 \%$ of the total ill infected persons. However, because the number of sick individuals is low, the emergence of drug resistance also remains at low levels. The whole epidemic runs at very low levels but lasts approximately as long as the original epidemic without any intervention.

Combined strategies can only be successful if the protective effect of prophylaxis and the reduction of susceptibility during treatment also apply to contacts between susceptibles and infecteds shedding resistant virus. 


\section{Summary}

The model we have developed gives a description of the impact that emergence of drug-resistant influenza strains may have during antiviral therapy or prophylaxis or both. The results of the model suggest the following:

- Treatment of only infected individuals developing clinical symptoms is associated with a high risk of developing drug resistance. In fact, the model predicts a strong emergence of drug-resistant virus causing a new epidemic within the original epidemic. This conclusion is strongly linked to our basic assumptions that sick infecteds can transmit the disease very rapidly, that individuals undergoing drug therapy have a high probability of shedding resistant virus, that the infectivity of drug-resistant viral strains is the same as that of the wild type virus, and that drug efficacy for prophylaxis and treatment is only partial. This behavior favors the emergence of drug-resistant virus since the small reduction in transmission maintains a relatively high level of infecteds providing the necessary time for drug-resistant strains to emerge and transmit.

- The spread of the epidemic and the degree of drug-resistant virus that develops in the model is higher than reported in closed population outbreaks $[2,3,5]$. Our model makes predictions in closer accord with experience if we assume that there is a difference in infectivity between wild type and drug-resistant strains. Under such conditions we find a modest beneficial effect to this treatment strategy.

- Using a strategy of prophylaxis leads to lower levels of infection but also to a prolongation of the epidemic. Emergence of drug resistance is also a problem. The reason again is our assumption that prophylaxis is not completely effective in preventing disease, and the infectivity of the drug-resistant virus is the same as that of the wild type. Thus, a person once infected has a high probability of shedding resistant virus. A low-infectivity assumption for the drug-resistant strains leads to a very strong blocking of the epidemic and very low emergence of drug resistant strains making this strategy superior to the previous one and worthy of recommendation.

- Combining treatment of infecteds with clinical symptoms and prophylaxis for susceptibles has effects that are similar to the first strategy. Intensive treatment and prophylaxis cause strong emergence of drug-resistant virus if there is no difference in infectivity between drug-resistant strains and wild type virus. This corresponds to transmission of resistant virus and associated failures of drug prophylaxis observed in household settings [1] where both treatment of ill index cases, particularly young children, and post contact prophylaxis are employed. Treatment and/or prophylaxis 
under the low-infectivity of drug-resistant variants assumption again shows beneficial effects.

\section{References}

1. Hayden, F.G., Belshe, R.B., Clover, R.D., Hay, A.J., Oakes, M.G., \& Soo, W. Emergence and apparent transmission of rimantadine-resistant influenza $A$ virus in families. N Engl J Med 1989; 321:1696-1702.

2. Mast, E.E., Harmon, M.W., Gravenstein, S., Wu, S.P., Arden, N.H., Circo, R., Tyszka, G., Kendal, A.P. \& Davis, J.P. Emergence and possible transmission of amantadine-resistant viruses during nursing home outbreaks of influenza A(H3N2). Amer $J$ Epidemiol 1991; 134:988-997.

3. Houck, P., Hemphill, M., LaCroix, S., Hirsh, D., \& Cox, N. Amantadine-resistant influenza A in nursing homes: identification of a resistant virus prior to drug use. Arch Int Med 1995; 155:533-537.

4. Hayden, F.G. Amantadine and rimantadine--clinical aspects. In: Richman D.D., ed Antiviral Drug Resistance. John Wiley \& Sons Ltd, 1996; 59-77.

5. Degelau, J., Somani, S.K., Cooper, S.L., Guay, D.R.P. \& Crossley, K.B. Amantadine-resistant influenza A in a nursing facility. Arch Intern Med 1992; 152:390-2.

6. Rose, H.J. The use of amantadine and influenza vaccine in a type A influenza epidemic in a boarding school. J $R$ Coll Gen Pract 1980; 30:619-21.

7. Oker-Blom, N., Hovi, T., Leinikki, P., Palosuo, T., Pettersson, R. \& Suni, J. Protection of man from natural infection with influenza A2 Hong Kong virus by amantadine: A controlled field trial. $\mathrm{Br} M e d J$ 1970; 3:676-8.

8. Nafta, I., Turcanu, A.G., Braun, I., Companetz, W., Simionescu, A., Birt, E. \& Florea, V. Administration of amantadine for the prevention of Hong Kong influenza. Bull World Health Org 1970; 42:423-7.

9. Monto, A.S., Gunn, R.A., Bandyk, M.G. \& King, C.L. Prevention of Russian influenza by amantadine. JAMA $1979 ; 241: 1003-7$.

10. Pettersson, R.F., Hellstrom, P.E., Penttinen, K., Phyala, R., Tokola, O., Vartio, T. \& Visakorpi, R. Evaluation of amantadine in the prophylaxis of influenza $A$ (H1N1) virus infection: A controlled field trial among young adults and high-risk patients. J Infect Dis. $1980 ; 142: 377-83$.

11. Quarles, J.M., Couch, R.B., Cate, T.R. \& Goswick, C.B., Comparison of amantadine and rimantadine for prevention of type A (russian) influenza. Antivita Res $1981 ; 1: 149-55$.

12. Ziegler, T., Hemphill, M., Ziegler, M.L., Klimov, A., \& Cox, N. Rimantadine resistance of influenza A viruses: An international surveillance. Presented at 7 th ISAR Conference, 1994 Feb 27--Mar 4, Charleston, South Carolina. 
13. Van Voris, L.P., Betts, R.F., Hayden, F.G., Christmas, W.A. \& Douglas, R.G., Jr. Successful treatment of naturally occuring influenza A/USSR/77 H1N1. JAMA 1981; 245:112-31.

14. Couch, R.B., Kasel, J.A., Glezen, W.P., Cate, T.R., Six, H.R., Taber, L.H., Frank, A.L., Greenberg, S.B., Zahradnik, J.M. \& Keitel, W.A. Influenza: Its control in persons and populations. J Infect Dis 1986; 153:431-40.

15. Bean, W.J., Threlkeld, S.C. \& Webster, R.G. Biologic potential of amantadineresistant influenza A virus in an avian model. $J$ Infect Dis 1989; 159:105-6.

16. Hayden, F.G., Sperber, S.J., Belshe, R.B., Clover, R.D., Hay, A.J., \& Pyke, S. Recovery of drug-resistant influenza A virus during therapeutic use of rimantadine. Antimicrob Agents Chemother 1991; 35:174-7.

17. Hall, C.B., Dolin, R., Gala, C.L., Markovitz, D.M., Zhang, Y.Q., Madore, P.H., Disney, F.A., Talpey, W.B., Green, J.L., Francis, A.B. \& Pichichero, M.E. Children with influenza A infection: treatment with remantadine. Pediatrics $1987 ; 80: 275-82$.

18.Rose, H.J. Use of amantadine in infuenza: a second report. $J$ R Coll Gen Pract 1983; 33:651-3.

19. Galbraith, A.W., Oxford, J.S., Schild, G.C. \& Watson, G.I. Protective effect of 1 adamantanamine hydrochloride on influenza $\mathrm{A} 2$ infections in the family environment. $\mathrm{A}$ controlled double-blind study. Lancet 1969; 2:1026-8.

20. Galbraith, A.W., Oxford, J.S., Schild, G.C. \& Watson, G.I. Study of 1-adamantanamine hydrochloride used prophylactically during the Hong Kong influenza epidemic in the family environment. Bull WHO 1969; 41:677-82. 


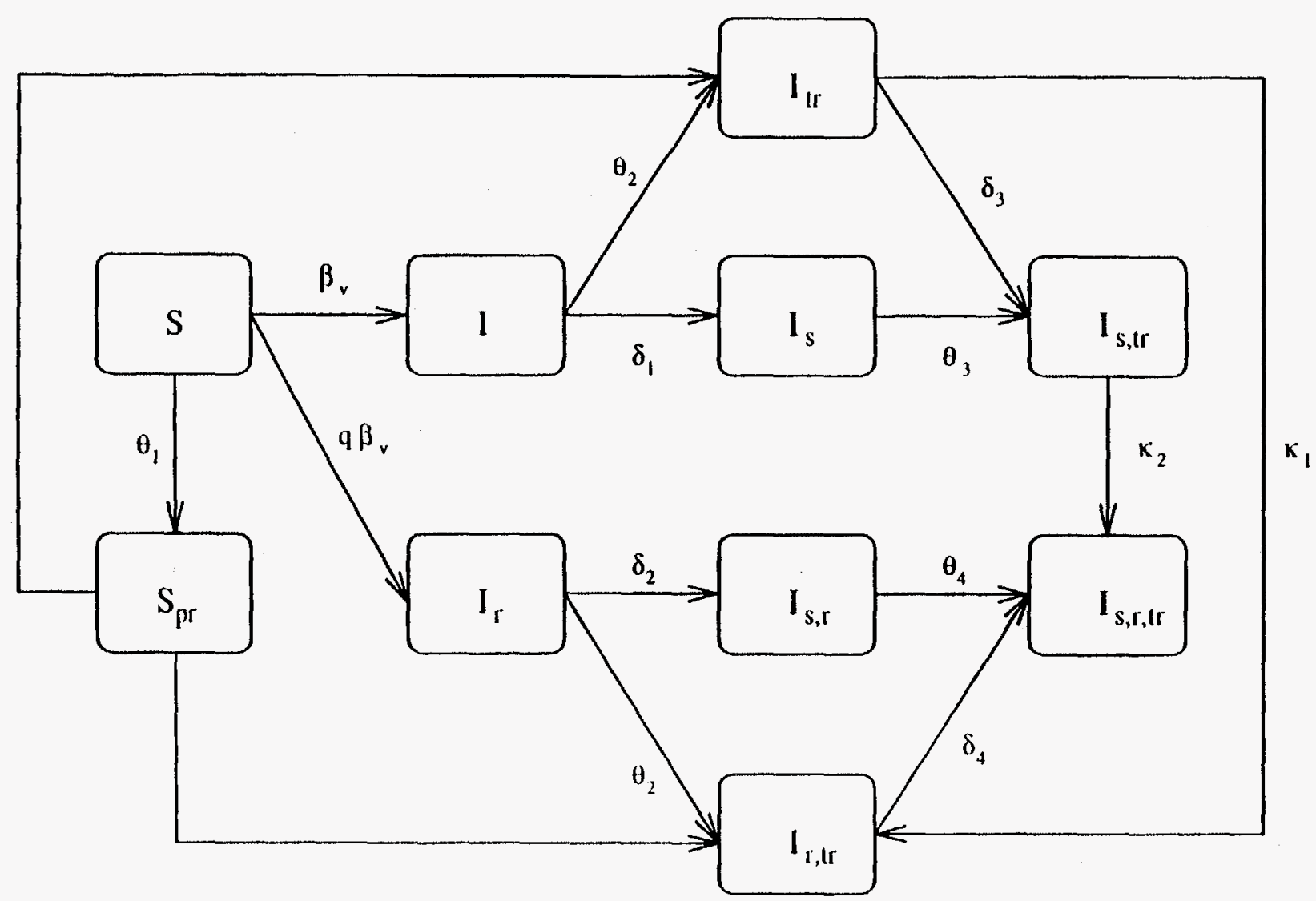

Figure 1. Schematic presentation of the model populations during treatment. Susceptibles $(S)$ become infected shedding wild type (I) or resistant virus (Ir). Susceptibles receiving prophylaxis, $S p r$, at rate $\theta_{1}$, become infected-treated shedding wild type, Itr, or resistant virus, $I_{r}$, tr. Infecteds from both groups develop clinical symptoms with rates $\delta 1$ and $\delta 2$, respectively. They can also receive treatment, if detected, with rate $\theta 2$. Subclinically infecteds whether shedding wild type, $I \mathbf{I r}$, or resistant virus, Ir,rr, receiving treatment can still become sick and continue receiving treatment with rates $\delta_{3}$ and $\delta 4$, respectively. Infecteds in both groups with clinical symptoms, $I_{s}, I_{r, s}$, receive treatment with rates $\theta_{3}$ and $\theta_{4}$. During treatment infecteds with clinical symptoms, Is,tr, develop drug resistance with a rate $\kappa 2$ and become infecteds treated shedding resistant virus, $I \mathrm{~s}, r, t r$. Drug resistance also develops from infecteds-treated with rate $\kappa$. All infecteds recover with rate $\delta \mathrm{m}$ and move to the pool of immunes (not shown). 


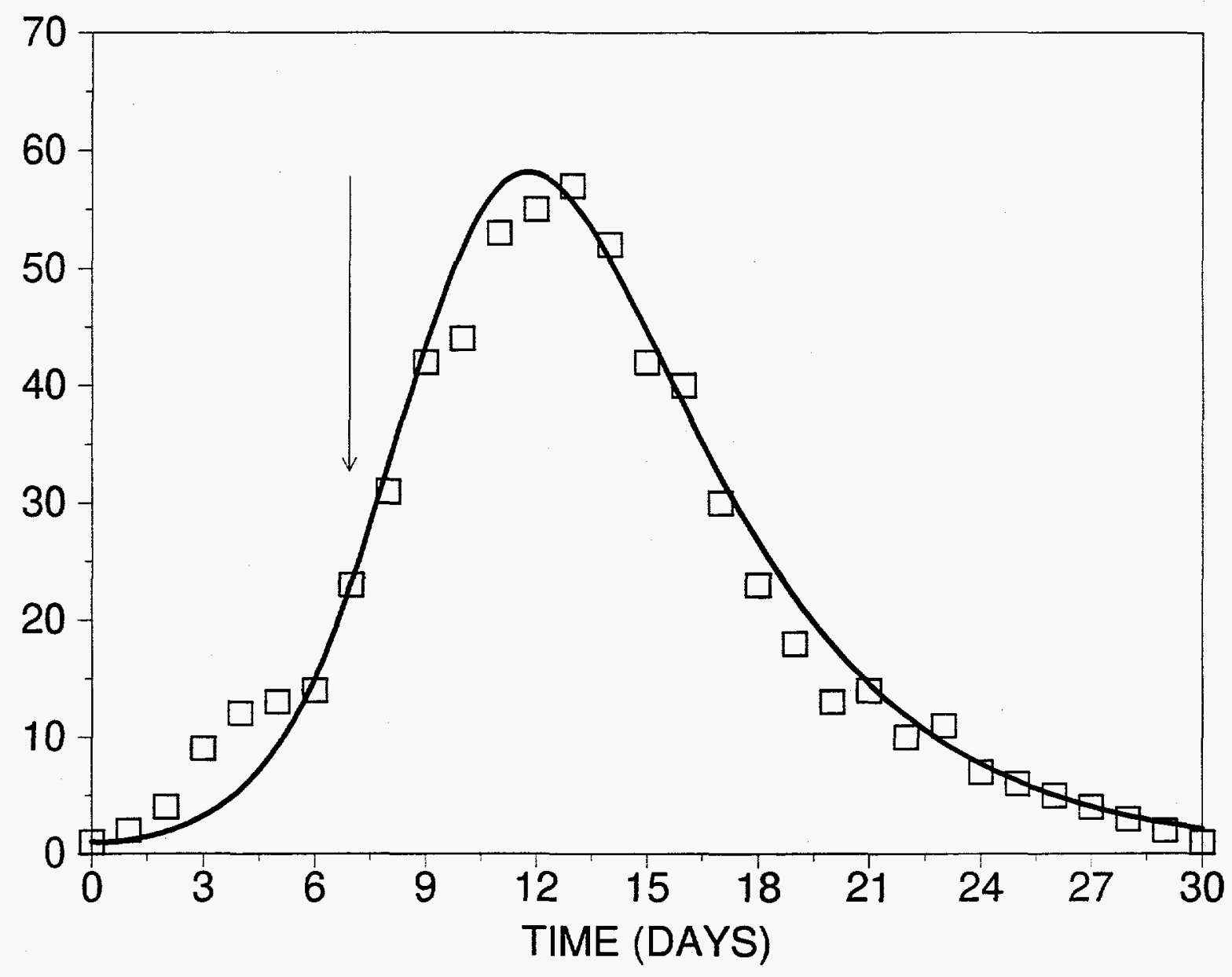

Figure 2. Fitting of data from an influenza epidemic in a boarding school. Parameter values: $\beta 1=0.0006 /$ day, $\beta 2=0.006 /$ day, $\delta 1=0.5 /$ day, $\gamma 1=0.5 /$ day and $\gamma_{2}=0.25 /$ day. Arrow indicates time at which prophylaxis is initiated. 
(a)

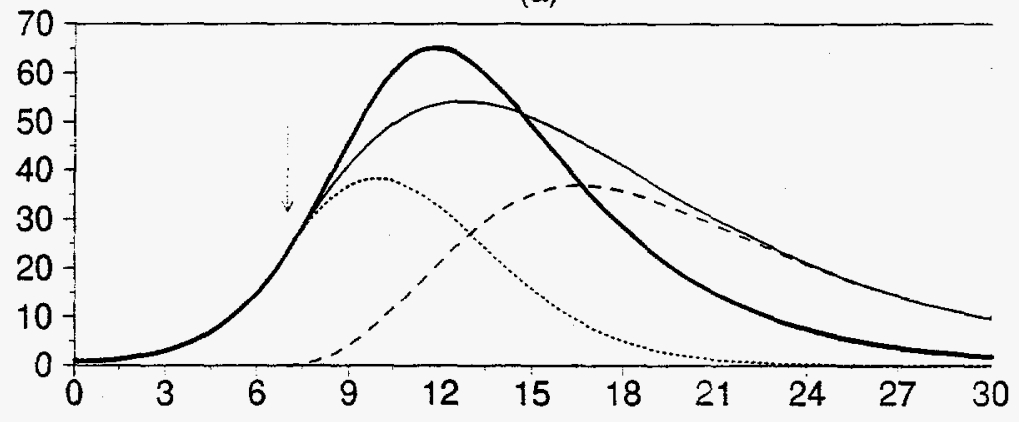

(b)

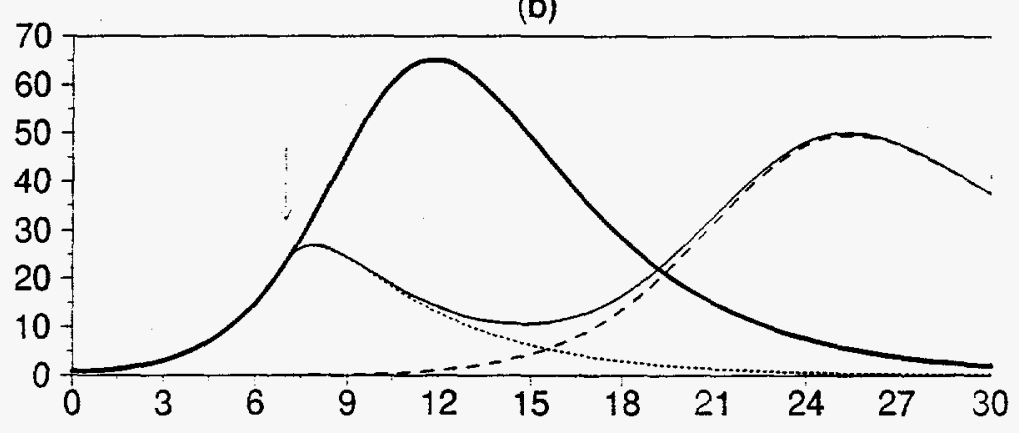

(c)

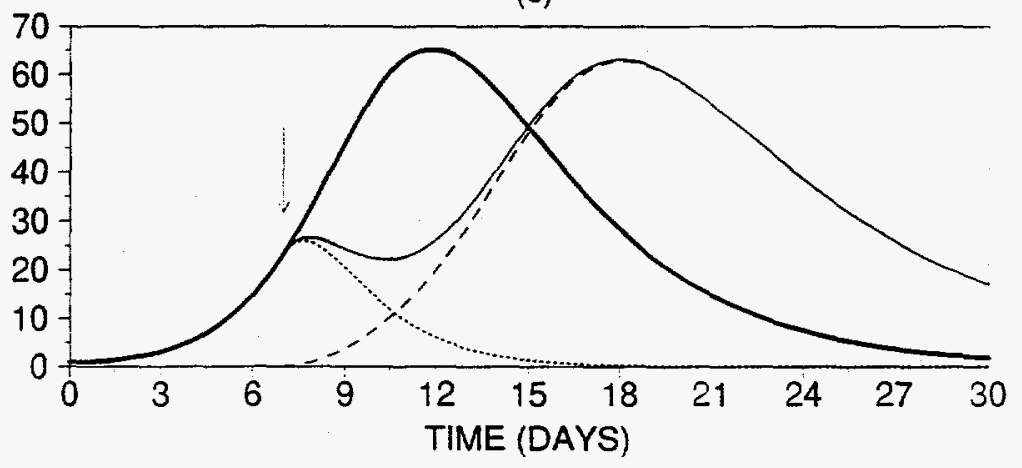

Figure 3. Treatment and/or prophylaxis during an epidemic introduced after 7 days (arrow) compared with the epidemic without any intervention (thick solid line), under the assumption that drug-resistant virus shows the same infectivity as wild type virus $(\mathrm{q}=1)$. a) Introduction of treatment of infected individuals showing clinical symptoms. Total number of infected individuals with clinical symptoms $I \mathrm{~s}+I \mathrm{~s}, t r+I \mathrm{~s}, r$ $+I s, r, t r$ (thin solid line); Number of infecteds shedding the wild type virus $I s+I s, t r$ (dotted line) and the population of those shedding resistant virus $I s, r+I s, r, t r$ (dashed line). b) Introduction of prophylaxis of all susceptibles instantaneously at day 7. Representation of populations as under a). c) Combined strategy of treatment and prophylaxis under the assumptions of $a$ ) and $b$ ). Representation of population as under a). Parameter values (per day) as in Fig. 2. In addition, $\gamma 4=\gamma 8=0.15, \gamma 5=1.00, \gamma 6=0.33, \delta 3=1 / 4$, $\kappa 1=0.1 \kappa 2, \kappa 2=0.25, \theta 3=\theta 4=0.5$. 
(a)

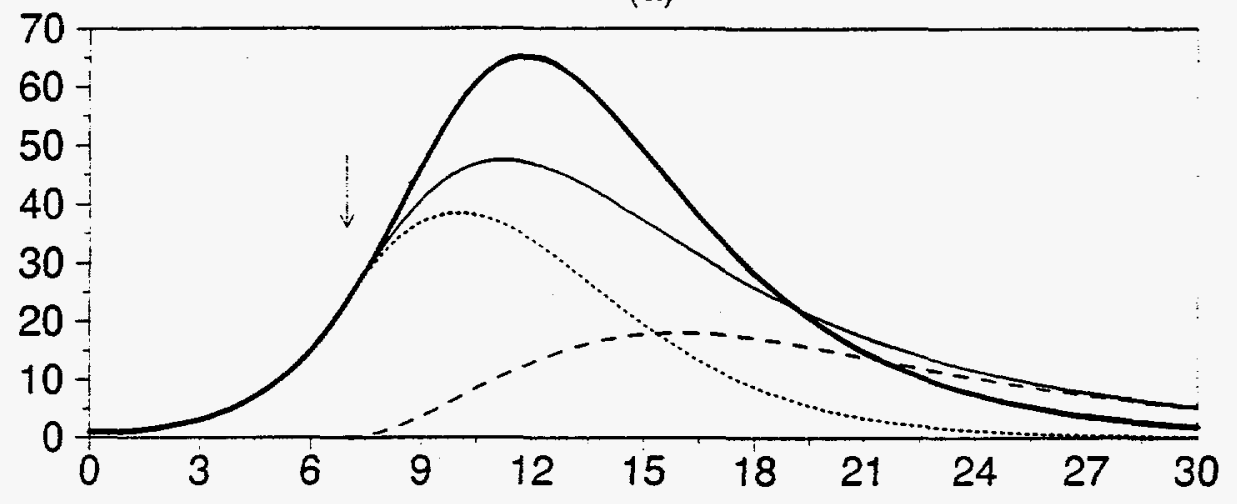

(b)

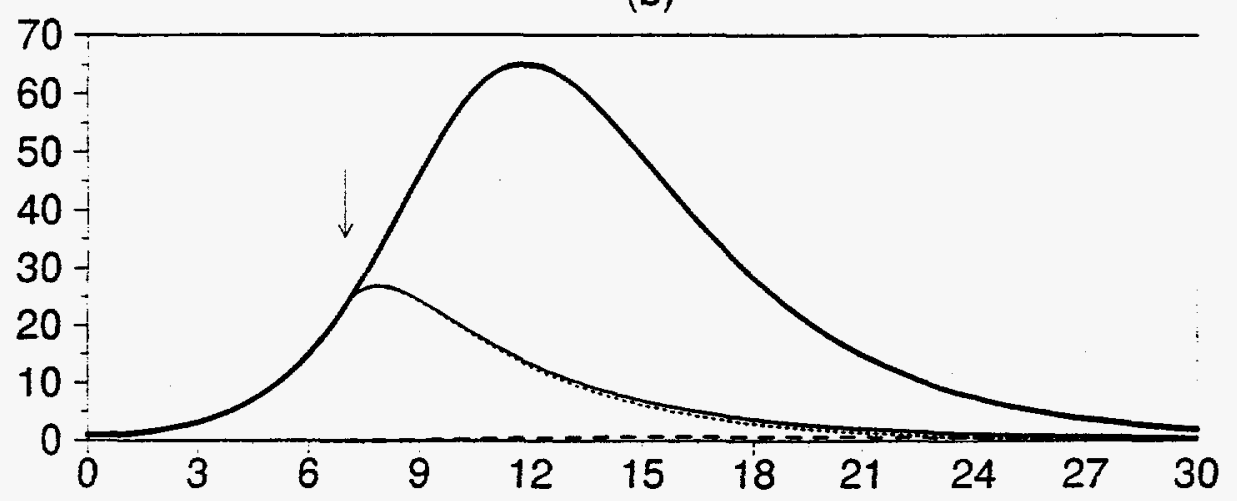

(c)

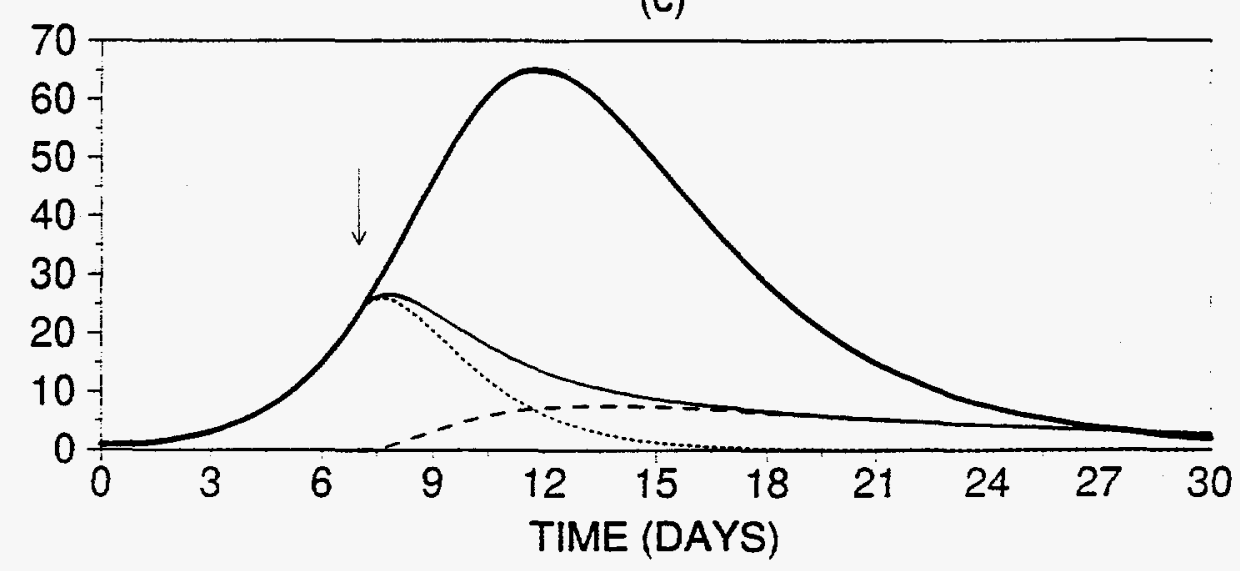

Figure 4. The same treatment and/or prophylaxis strategies as in Fig. 3 assuming a 10-fold lower infectivity of drug-resistant strains. 